\title{
A NOTE ON MAXIMAL AND MINIMAL CAUSAL SPACES
}

\author{
SRIBATSA NANDA
}

(Received 8 November 1978)

\begin{abstract}
It is shown in this note that the supremum and infimum of all causal topologies on Minkowski space are not causal. It is further shown that maximal and minimal elements exist in the set of all principal causal spaces.
\end{abstract}

\section{Introduction}

Following the work of Zeeman [9], several authors [1-6] have suggested new topologies on Minkowski space, the four-dimensional space-time continuum of Special Relativity. All these topologies are characterized by the property that they have the same homeomorphism group $G$, the group generated by the inhomogeneous Lorentz group and dilatations (multiplication by positive scalars). Since every element of $G$ either preserves or reverses the causal relation on Minkowski space, these topologies are usually referred to as causal topologies. Some authors have also suggested topologies (see, for sample, [7]) whose homeomorphism groups are all equal to $G_{0}$, the subgroup of $G$ consisting of all elements which preserve the causal relation. It is well known that this subgroup $G_{0}$ is most important from a physical point of view; we shall, therefore, refer to these topologies (that is, the ones having homeomorphism group $G_{0}$ ) as causal topologies. A large number of causal topologies are now known and there is no reason why any one of them should be preferred to another (for both satisfy the same property). Ordinarily, one would be led to believe that the supremum and the infimum of the causal topologies would be of interest. Alternatively, one would look for maximal or minimal elements in the set of all causal topologies. It turns out, however, that the supremum and the infimum of all the causal topologies are not causal. The aim of this note is to prove these and other related results. 


\section{Notation and terminology}

Let $M$ denote Minkowski space with characteristic quadratic form $Q$ :

Let

$$
\begin{aligned}
M & =\left\{\left(x_{0}, x_{1}, x_{2}, x_{3}\right): x_{i} \text { are reals }\right\}, \\
Q(x) & =x_{0}^{2}-x_{1}^{2}-x_{2}^{2}-x_{3}^{2} .
\end{aligned}
$$

$K=\left\{x \in M: Q(x)>0\right.$ and $\left.x_{0}>0\right\}$

and $L=\left\{x \in M: Q(x) \geqslant 0\right.$ and $\left.x_{0}>0\right\}$.

Define $K^{*}=K \cup\{0\}$ and $L^{*}=L \cup\{0\}$. Denote by $K^{*}(x)$ and $L^{*}(x)$, the translates of $K^{*}$ and $L^{*}$ respectively: $K^{*}(x)=K^{*}+x$ and $L^{*}(x)=L^{*}+x$. We shall (by an abuse of terminology) write $-K^{*}(x)$ and $-L^{*}(x)$ for the sets $-K^{*}+x$ and $-L^{*}+x$ respectively. We have the following cones at $x$ :

light cone at $x: \quad C^{L}(x)=\{y \in M: Q(y-x)=0\} \cup\{x\} ;$

time cone at $x: \quad C^{T}(x)=\{y \in M: Q(y-x)>0\} \cup\{x\}$;

space cone at $x: C^{S}(x)=\{y \in M: Q(y-x)<0\} \cup\{x\}$.

Note that $C^{T}(x)=C_{+}^{T}(x) \cup C_{-}^{T}(x)$, where

$$
C_{+}^{T}(x)=K^{*}+x \text { and } C_{-}^{T}(x)=-K^{*}+x .
$$

Similarly, $C^{L}(x)=C_{+}^{L}(x) \cup C_{-}^{L}(x)$, where $C_{+}^{L}(x)$ and $C_{-}^{L}(x)$ are the forward and backward light cones at $x$. The causality relation in $M$ is represented by a partial order $<$ defined by

$$
x<y \Leftrightarrow y-x \in K .
$$

We shall denote by $d$ the usual Euclidean distance function on $M$, that is

$$
d(x, y)=\left\{\sum_{i=0}^{3}\left(x_{i}-y_{i}\right)^{2}\right\}^{\frac{1}{2}},
$$

and by $N_{\varepsilon}^{E}(x)$, a Euclidean neighbourhood of radius $\varepsilon$ about $x$, that is

$$
N_{\varepsilon}^{E_{i}}(x)=\{y \in M: d(x, y)<\varepsilon\} .
$$

\section{The supremum and the infimum}

As pointed out in the Introduction, the supremum and the infimum of all causal topologies are not causal. To prove this, we need two known causal topologies, namely the $s$-topology and the $t$-topology [2].

Let $\varepsilon>0$ and $N_{\varepsilon}^{t}(x)=N_{\varepsilon}^{E}(x) \cap C^{T}(x)$. The $t$-topology on $M$ is defined to be the topology generated by taking $\left\{N_{\varepsilon}^{t}(x): x \in M, \varepsilon>0\right\}$ as a basis; alternatively, we may take $\left\{N_{\varepsilon}^{t}(x): x\right.$ has rational coordinates, $\varepsilon$ rational $\}$ as a basis for the same 
topology, thus making it second countable. Similarly, let $N_{\varepsilon}^{s}(x)=N_{\varepsilon}^{E}(x) \cap C^{S}(x)$; then the $s$-topology on $M$ is defined to be the topology generated by taking $\left\{N_{\varepsilon}^{s}(x): x \in M, \varepsilon>0\right\}$ as a basis. The $s$-topology is also second countable.

Proposition 3.1. The supremum of all the causal topologies on $M$ is not causal.

Proof. Consider $s \vee t$, the supremum of the $s$-topology and the $t$-topology. For any $x \in M$ and $\varepsilon>0, N_{\varepsilon}^{s}(x)$ and $N_{\varepsilon}^{t}(x)$ are open in $s \vee t$ so is $N_{\varepsilon}^{s}(x) \cap N_{\varepsilon}^{t}(x)=\{x\}$. Thus $s \vee t$ is the discrete topology. The supremum of all the causal topologies, being finer than $s \vee t$, is therefore discrete and it cannot be causal.

Recall from [4] that every first countable Hausdorff topology $T$ on a space $X$ has an anti-topology $T^{*}$ which has the same group of homeomorphisms as that of $T$. The anti-topology $T^{*}$ is characterized by the property that its closed sets (except $X$ ) are precisely the compact sets of $T$. Thus the closed sets of $t^{*}$ are those which are closed and bounded subsets on time-like lines of $M$ or their finite unions; similarly, the closed subsets of $s^{*}$ are those which are closed and bounded subsets on space-like hyperplanes or their finite unions. We are now ready to prove

\section{Proposition 3.2. The infimum of all the causal topologies on $M$ is not causal.}

Proof. Consider $s^{*} \wedge t^{*}$, the infimum of the topologies $s^{*}$ and $t^{*}$. Let $U(\neq M)$ be open in $s^{*} \wedge t^{*}$. Since $U$ is open in $t^{*}$, it must be of the form $A^{c}$, where $A$ is a closed and bounded subset (in the Euclidean sense) lying on a finite number of time-like lines, and $c$ denotes the complement. Similarly, since $U$ is open in $s^{*}$, it must be of the form $B^{c}$, where $B$ is a closed and bounded set (in the Euclidean sense) lying on a finite number of space-like hyperplanes. This is possible if and only if $A(=B)$ is a (discrete) finite set of points. Thus $s^{*} \wedge t^{*}$ is the co-finite topology on $M$. If $T$ denotes the infimum of all the causal topologies, then it is clear that $T$ is weaker than $s^{*} \wedge t^{*}$. We shall show that $T$ is either (i) the indiscrete topology or (ii) the co-finite topology.

Suppose that $T$ is not the indiscrete topology; then there is an open set $U \neq M$ whose complement is finite. Let $U^{c}=\left\{x_{1}, x_{2}, \ldots, x_{n}\right\}$. Note that any element $g \in G_{0}$ is a homeomorphism in a causal topology, so that it is a homeomorphism in $T$. We can therefore make a translation through $-x_{1}$ to get a set

$$
V=\left\{0, x_{2}-x_{1}, \ldots, x_{n}-x_{1}\right\}
$$

which is closed in $T$. Apply a rotation $g \in G_{0}$ to get a closed set

$$
g V=\left\{0, g x_{2}-g x_{1}, \ldots, g x_{n}-g x_{1}\right\}
$$

the choice of the element $g$ is so made that there are no common elements in the sets of non-zero elements of $V$ and $g V$. Thus $V \cap g V=\{0\}$, showing that $\{0\}$ is 
a closed set in $T$. Since translations are homeomorphisms in $T$, every singleton is closed in $T$, and so are the finite sets; thus $T$ must be the cofinite topology.

In either case, any bijective map of $M$ onto itself is a homeomorphism and the topology cannot be causal. This completes the proof of the Proposition.

Instead of considering the supremum and the infimum of all causal topologies, we can consider the supremum and the infimum of all causal topologies on $M$ having a particular property, and they may turn out to be causal. Indeed, Zeeman's fine topology is characterized by the property that, in the set of all causal spaces which induce the one-dimensional Euclidean topology on time-like lines and the three-dimensional Euclidean topology on space-like hyperplanes, the fine topology is the supremum. Similarly, the $A$-topology [3] can be characterized as the supremum of all causal spaces having a certain property.

\section{Maximal and minimal elements}

Since the supremum and the infimum of all the causal topologies are not causal, one would next try to look for maximal or minimal elements in the set of all causal spaces. The following results are easy to derive and we omit their proofs.

Proposition 4.1. Let $\left\{T_{i}\right\}_{i \in I}$ be a set of topologies on $M$ each having the homeomorphism group $G$ and let $H$ be the homeomorphism group of $\inf _{i \in I} T_{i}$; then $G \subset H$.

Proposition 4.2. Let $\left\{T_{i}\right\}_{i \in I}$ be a set of topologies on $M$ each having the homeomorphism group $G$ and let $H$ be the homeomorphism group of $\sup _{i \in I} T_{i}$ then $G \subset H$.

REMARK 4.3. Propositions 4.1 and 4.2 are valid if $G$ is replaced by $G_{0}$.

The difficult step in proving the existence of maximal (or minimal) elements is to start with a linearly ordered set of topologies $\left\{T_{i}\right\}_{i \in I}$ and to prove that $H \subset G$. There is no reason to believe that this can be done. Our conjecture is that maximal (or minimal) elements do not exist in the set of causal spaces. To prove this, however, one has to construct, starting with a given topology, a strictly finer (or weaker) topology which is also causal.

The problem of finding a maximal or minimal causal space can be slightiy weakened as follows. Consider (see [5]) the order-topology on $M$ which is generated by taking the family of positive cones $\left\{K^{*}(x)\right\}_{x \in M}$ as a basis. We shall call this the $K$-topology. Similarly, we define the $L$-topology on $M$ to be the topology generated by taking the cones $\left\{L^{*}(x)\right\}_{x \in M}$ as a basis. It is known that 
both these topologies are causal with homeomorphism group $G_{0}$. A notable feature of these two topologies is that they are principal. (A topology $T$ on $X$ is said to be principal if each point $x \in X$ has a smallest $T$-neighbourhood $B_{T}(x)$.) We shall show that, in the set of all principal causal topologies on $M$, the $K$ topology is a maximal element and the $L$-topology is a minimal element. We need the following lemmas.

Lemma 4.4. Let $T_{1}$ and $T_{2}$ be two principal topologies on $M$ with $T_{1}<T_{2}$; then $B_{T_{1}}(x) \supset B_{T_{2}}(x)$ for every $x \in M$.

Proof. Since $B_{T_{1}}(x)$ is an open set in $T_{1}$ containing $x$, it is also open in $T_{2}$ (since $\left.T_{1}<T_{2}\right)$ and therefore includes the smallest open set $B_{T_{2}}(x)$ about $x$. Thus the proof is complete.

Lemma 4.5. Let $T$ be a principal topology on $M$ and let $h$ be a homeomorphism of $(M, T)$. Then $h\left(B_{T}(x)\right)=B_{T}(h x)$.

Proof. As $B_{T}(h x)$ is open, $h^{-1}\left(B_{T}(h x)\right)$ is open about $x$ and therefore contains the smallest open set $B_{T}(x)$. Thus $B_{T}(x) \subset h^{-1}\left(B_{T}(h x)\right)$ or, equivalently, $h\left(B_{T}(x)\right) \subset B_{T}(h x)$. Similarly, since $h$ is open, $h\left(B_{T}(x)\right) \supset B_{T}(h x)$. The result therefore follows.

Proposition 4.6. The $K$-topology is maximal in the set of all principal causal spaces on $M$.

Proof. Assume to the contrary that there exists a principal causal topology $T$ on $M$ which is strictly finer than the $K$-topology. Note that, for the $K$-topology, the smallest open set $B_{K}(x)$ about $x$ is just $K^{*}(x)$; so, by Lemma 4.4, we have $B_{T}(x) \subset K^{*}(x)$. We claim that $B_{T}(x) \neq K^{*}(x)$ for every $x \in M$; for, if $B_{T}(x)=K^{*}(x)$ for some $x$, then we can make a translation of these sets to get $B_{T}(y)=K^{*}(y)$ for every $y \in M$ (since by our assumption every translation is a homeomorphism for each of these topologies $K$ and $T$ ), thus making these topologies equal. So $K^{*}(x)-B_{T}(x) \neq \varnothing$.

A translation gives us $K^{*}-B_{T}(0) \neq \varnothing$. Let $z \in K^{*}-B_{T}(0)$. Since every dilatation is a homeomorphism of the topology $T$, it follows that $k z \notin\left\{k \cdot B_{T}(0)\right\}=B_{T}(0)$ (by Lemma 4.5). Thus the entire half-line joining the origin and the point $z$ lies in $K^{*}$ but outside $B_{T}(0)$. Choose a point $p \in B_{T}(0)$, a point $q$ in the half-line mentioned above and a rotation $g \in G_{0}$ such that $g(q)=p$. Since $g$ is a homeomorphism of both the topologies, we have

$$
g\left(B_{T}(0)\right)=B_{T}(0) \text { and } g\left(K^{*}\right)=K^{*}
$$


Consequently, $g\left(K^{*}-B_{T}(0)\right)=K^{*}-B_{T}(0)$. Thus $p=g(q) \in K^{*}-B_{T}(0)$ which is a contradiction since $p \in B_{T}(0)$. This completes the proof of the Proposition.

We need slightly different arguments to prove the following

Proposition 4.7. The L-topology is minimal in the set of all principal causal topologies on $M$.

Proof. Let $T$ be a principal causal topology on $M$ such that $T$ is strictly weaker than $L$. Lemma 4.4 then implies that, for any $x \in M, B_{T}(x) \supset L^{*}(x)$. We can then repeat the introductory arguments of Proposition 4.6 above to conclude that, for each $x \in M, B_{T}(x) \neq L^{*}(x)$; thus, $B_{T}(x)-L^{*}(x) \neq \varnothing$ or, equivalently,

$$
B_{T}(0)-L^{*} \neq \varnothing \text {. }
$$

Let $z_{1} \in B_{T}(0)-L^{*}$.

There are now three possibilities: (i) $z_{1}$ is space-like; (ii) $z_{1}$ is on the backward null cone at the origin; (iii) $z_{1}$ is on the backward time cone at the origin. We shall show that each of these cases will give rise to a contradiction.

Consider case (i) and suppose that $z_{1}$ is space-like. Let $z$ be any space-like vector; we can then choose a suitable rotation $g \in G_{0}$ such that $g z_{1}=z$. Since $z_{1} \in B_{T}(0)-L^{*}, z=g z_{1} \in g\left(B_{T}(0)\right)-g L^{*}=B_{T}(0)-L^{*}$, showing that the whole space cone at the origin $C^{S}(0) \subset B_{T}(0)-L^{*}$. Equivalently,

$$
C^{S}(0) \cup L^{*} \subset B_{T}(0) \text {. }
$$

We claim that this inclusion is strict; for, if these sets are assumed to be equal, that is

$$
B_{T}(0)=C^{S}(0) \cup L^{*}=\left(-L^{*}\right)^{c},
$$

then, for any $y \in M$, we can make a translation to get

$$
B_{T}(y)=C^{S}(y) \cup L^{*}(y)=\left(-L^{*}(y)\right)^{c} .
$$

Let $y_{1}$ and $y_{2}$ be two points such that $y_{2}-y_{1}$ is space-like; then

$$
B_{T}\left(y_{1}\right) \cap B_{T}\left(y_{2}\right)=\left[\left(-L^{*}\left(y_{1}\right)\right)-\left(-L^{*}\left(y_{2}\right)\right)\right]^{c} .
$$

If we take $y=\frac{1}{2}\left(y_{1}+y_{2}\right)$, then it is obvious that $y \in B_{T}\left(y_{1}\right) \cap B_{T}\left(y_{2}\right)$ whereas $B_{T}(y) \notin B_{T}\left(y_{1}\right) \cap B_{T}\left(y_{2}\right)$. This is a contradiction so that our claim is proved.

There is, therefore, at least one point $z_{2} \in B_{r}(0)-\left(C^{S}(0) \cup L^{*}\right)$. There are now two possibilities. Either (a) $z_{2}$ is on the backward null cone at the origin or (b) $z_{2}$ is on the backward time cone at the origin. Suppose that (a) holds; then for any $z$ on the backward light cone at the origin we can choose a $g \in G_{0}$ such that $g z_{2}=z$. 
Thus

$$
z=g z_{2} \in g\left(B_{T}(0)\right)-\left(g C^{S}(0) \cup L^{*}\right)=B_{T}(0)-\left(C^{S}(0) \cup L^{*}\right),
$$

showing that the entire backward null cone at the origin

$$
C_{-}^{L}(0) \subset B_{T}(0)-\left(C^{S}(0) \cup L^{*}\right) .
$$

We claim again that this inclusion is strict; for, if the two sets are equal, then

$$
B_{T}(0)=C^{s}(0) \cup L^{*} \cup C_{-}^{L}(0)=\left(-K^{*}\right)^{c}
$$

and, through a translation, we get

$$
B_{T}(x)=\left[-K^{*}(x)\right]^{c},
$$

which is true for any $x \in M$. As before, we can take two points $x_{1}$ and $x_{2}$ so that $x_{2}-x_{1}$ is space-like, so that

$$
B_{T}\left(x_{1}\right) \cap B_{T}\left(x_{2}\right)=\left[\left(-K^{*}\left(x_{1}\right)\right) \cup\left(-K^{*}\left(x_{2}\right)\right)\right]^{c} .
$$

Taking $x_{1}=\frac{1}{2}\left(x_{1}+x_{2}\right)$, we find that $x \in B_{T}\left(x_{1}\right) \cap B_{T}\left(x_{2}\right)$ but

$$
B_{T}(x) \oplus B_{T}\left(x_{1}\right) \cap B_{T}\left(x_{2}\right) ;
$$

hence we have a contradiction. Thus strict inequality holds and we have at least one point $z_{3} \in B_{T}(0)-\left(C^{S}(0) \cup L^{*} \cup C_{-}^{L}(0)\right)$. Clearly $z_{3}$ is on the backward time cone at the origin. We can then choose a rotation $g \in G_{0}$ so that $g z_{3}$ is any other backward-pointing time-like vector; thus

$$
C_{-}^{T}(0) \subset B_{T}(0)-\left(C^{S}(0) \cup L^{*} \cup C_{-}^{L}(0)\right)
$$

or, alternatively, $M \subset B_{T}(0)$; but, since $B_{T}(0)$ is a subset of $M$, it follows that $M=B_{T}(0)$. The topology $T$ is therefore indiscrete and it cannot be causal and we have a contradiction.

In the case when (b) holds, we have a $z_{2}$ on the backward time cone at the origin. We can then proceed similarly as in case (a) to show that $B_{T}(0)=M$. We have thus shown that possibility (i) gives us a contradiction. We omit the proof that possibilities (ii) and (iii) will also give us contradictions (the line of proof is the same). Thus our assumption that there exists a principal topology strictly weaker than $L$ is false, and this completes the proof of the Proposition.

Principal spaces, however, are simple in nature and, for this reason, it has been possible to derive Propositions 4.6 and 4.7 with considerable ease. Whether maximal and minimal elements exist in the set of all causal topologies is now an open question. 


\section{References}

[1] S. Nanda, "Topology for Minkowski space", J. Math. Phys. 12 (1971), 394-401.

[2] S. Nanda, "Weaker versions of Zeeman's conjectures on topologies for Minkowski space", J. Math. Phys. 13 (1972), 12-15.

[3] S. Nanda, "A-topology for Minkowski space", J. Austral. Math. Soc. B 21 (1979), 53-64.

[4] S. Nanda and H. K. Panda, "Compact topologies for Minkowski space", Int. J. Theor. Phys. 10 (1974), 159-163.

[5] S. Nanda and H. K. Panda, "Minkowski space with order topology is simply connected", Int. J. Theor. Phys. 12 (1975), 393-399.

[6] P. G. Vroegindeweij, (Ph.D. Thesis, Rijks University, Domplein, Utrecht, 1973).

[7] G. S. Whiston, "The Zeeman order topology on Minkowski space", Int. J. Theor. Phys. 6 (1972), 75-77.

[8] G. Williams, "Fine topologies for Minkowski space", Proc. Camb. Phil. Soc. 76 (1974), 503-510.

[9] E. C. Zeeman, "Topology for Minkowski space”, Topology 6 (1967), 161-170.

\section{Mathematics Department}

Regional Engineering College

Rourkela-769008 (Orissa)

India 